\title{
S-process in extremely metal-poor, low-mass stars
}

\author{
M. A. Cruz ${ }^{1}$, A. Serenelli2,1, and A. Weiss ${ }^{1}$ \\ 1 Max-Planck Institut für Astrophysik, Karl-Schwarzschild-Str. 1, 85748 Garching bei München, Germany \\ e-mail: aldos@ice.csic.es \\ ${ }^{2}$ Instituto de Ciencias del Espacio (CSIC-IEEC), Facultad de Ciències, Campus UAB, 08193 Bellaterra, Spain \\ Received 30 April 2012 / Accepted 19 August 2013
}

\begin{abstract}
Context. Extremely metal-poor (EMP), low-mass stars experience an ingestion of protons into the helium-rich layer during the core He-flash, resulting in the production of neutrons through the reactions ${ }^{12} \mathrm{C}(\mathrm{p}, \gamma){ }^{13} \mathrm{~N}(\beta){ }^{13} \mathrm{C}(\alpha, \mathrm{n}){ }^{16} \mathrm{O}$. This is a potential site for the production of s-process elements in EMP stars, which does not occur in more metal-rich counterparts. The signatures of s-process elements in the two most iron deficient stars observed to date, HE1327-2326 \& HE0107-5240, still await for an explanation.

Aims. We investigate the possibility that low-mass EMP stars could be the source of s-process elements observed in extremely iron deficient stars, either as a result of self-enrichment or in a binary scenario as the consequence of a mass transfer episode.

Methods. We present evolutionary and post-processing s-process calculations of a $1 M_{\odot}$ stellar model with metallicities of $Z=0,10^{-8}$, and $10^{-7}$. We assess the sensitivity of nucleosynthesis results to uncertainties in the input physics of the stellar models with particular regard to the details of convective mixing during the core He-flash.

Results. Our models provide the possibility of explaining the $\mathrm{C}, \mathrm{O}, \mathrm{Sr}$, and $\mathrm{Ba}$ abundance for the star HE0107-5240 as the result of mass-transfer from a low-mass EMP star. The drawback of our model is that nitrogen would be overproduced and the ${ }^{12} \mathrm{C} /{ }^{13} \mathrm{C}$ abundance ratio would be underproduced in comparison to the observed values if mass would be transferred before the primary star enters the asymptotic giant branch phase.

Conclusions. Our results show that low-mass EMP stars cannot be ruled out as companion stars that might have polluted HE1327-2326 and HE0107-5240 and produced the observed s-process pattern. However, more detailed studies of the core He-flash and the proton ingestion episode are needed to determine the robustness of our predictions.
\end{abstract}

Key words. stars: evolution - nuclear reactions, nucleosynthesis, abundances - stars: individual: HE1327-2326 stars: individual: HE0107-5240 - stars: abundances

\section{Introduction}

Extremely metal-poor (EMP; $[\mathrm{Fe} / \mathrm{H}]<-3.0$ - Beers \& Christlieb 2005) stars are an important key for understanding galactic chemical evolution during the early stages of our Galaxy. In addition, abundance patterns of EMP stars might shed light on individual nucleosynthesis processes, contrary to those of more metal-rich stars, which reflect the well-mixed products of several nucleosynthesis processes in multiple generations of stars. The abundance patterns of EMP stars observed with high resolution spectroscopy are peculiar. A most conspicuous finding is that the fraction of EMP stars that are carbon enhanced ([C/Fe] > 0.9; Masseron et al. 2010) is 20\% (Rossi et al. 1999; Suda et al. 2011), which is much larger than the $\sim 1 \%$ found in more metal-rich stars (Tomkin et al. 1989; Luck \& Bond 1991). In terms of neutron capture elements, EMP stars also have a wide variety of patterns with stars showing no signatures of these elements to others having large enhancements in either s-process, r-process elements, or both (Beers \& Christlieb 2005). Moreover, the majority of carbon-enhanced EMP (CEMP) stars are also enriched in s-process elements, suggesting a common origin for carbon and s-process elements in these stars.

Star formation theory can also benefit from the study of abundance patters of EMP stars. The longstanding argument that primordial stars were extremely massive $\left(M \sim 100 M_{\odot}\right)$ and isolated systems (Abel et al. 2002; Bromm et al. 2002; Bromm \& Loeb 2004; O'shea \& Norman 2007; Yoshida et al. 2008) is being confronted by new numerical simulations of Population III star formation. Simulations by Greif et al. (2011) seem to indicate that Pop. III stars can be formed in multiple systems with a flat protostellar mass function $\left(M \sim 0.1-10 M_{\odot}\right)$. Moreover, the recent discovery of an EMP star with a metallicity of $[\mathrm{Fe} / \mathrm{H}]=$ -4.89 and no enhancement in CNO elements (Caffau et al. 2011, 2012), which results in the lowest total metallicity $Z \leq 10^{-4} Z_{\odot}$ observed in a star, also puts into question the claims of a minimum metallicity required for the formation of low-mass stars (Bromm \& Loeb 2003; Schneider et al. 2003). These results reinforce the necessity of determining the mass range of the stars responsible for the chemical signatures that are observed in EMP stars to constrain the initial mass function in the early Universe.

Several attempts have been made to explain the peculiarities of EMP abundance patterns; their origin, nevertheless, is still an unsolved puzzle. One of the first scenarios proposed to explain the overabundance of carbon and nitrogen in EMP stars involved the ingestion of protons into the helium convective zone (proton ingestion episode, hereafler PIE) that happens during the core He-flash in Pop. III and EMP stars (Fujimoto et al. 1990; Schlattl et al. 2001; Weiss et al. 2004; Picardi et al. 2004). The proton ingestion into the helium-rich convective layers during the core He-flash is a robust phenomenon in one dimensional stellar evolution calculations of low metallicity stars $([\mathrm{Fe} / \mathrm{H}]<-4.5)$ that subsequently leads to a large surface enrichment in CNO-elements (Hollowell et al. 1990; Schlattl et al. 2001; Campbell \& Lattanzio 2008). However, the amount of C, $\mathrm{N}$, and $\mathrm{O}$ dredged-up to the surface in the models is usually too large (1 to 3 dex larger than the observed $[\mathrm{C} / \mathrm{Fe}])$ to match the 
observations, disfavoring the self-enrichment scenario. In addition, most observed EMP stars are not evolved past the heliumcore flash to have undergone PIE.

The positive correlation between carbon and barium abundances observed in EMP stars indicates that carbon and s-process elements might have a common production site (Aoki et al. 2002; Suda et al. 2004, 2011). Stars with a metallicity of $[\mathrm{Fe} / \mathrm{H}] \leq-2.5$ in the mass range of $1.2 M_{\odot}<M \leq 3.0 M_{\odot}$ also experience a PIE during the first one or two thermal pulses in the beginning of the thermally pulsating asymptotic giant branch (TP-AGB) phase. Similar to the occurrence of the PIE during the core He-flash, proton-rich material is dragged down into the He-burning zone during the development of a thermal runaway in the He-shell. After the ensuing dredge-up event, the stellar envelope is strongly enriched in CNO elements (and possibly s-process). An alternative scenario, based on the previous considerations, was proposed by Suda et al. (2004). They argued that this is the most likely production environment for carbon and s-process elements. The observed abundances would be, then, the result of mass transfer from an AGB companion (now a white dwarf). Radial-velocity monitoring has shown that a large fraction of the CEMP-s (the subset of CEMP stars enriched in s-process elements) presents variable radial velocities, strengthening the argument of a binary scenario for the formation of CEMP-s stars (Lucatello et al. 2005).

Assuming that carbon and s-process enhancements in CEMP-s stars are the result of mass-transfer from a primary star, the question remains whether the donor stars are low- or intermediate-mass stars. Suda et al. (2004) claim that intermediate-mass stars are the polluting stars, which are responsible for the observed enhancements in carbon and s-process elements in CEMP-s stars. Campbell et al. (2010) have calculated models of low-mass EMP stars during the core He-flash and found that the PIE leads to the production of a large amount of neutrons $\left(n_{\mathrm{n}}>10^{14} \mathrm{~cm}^{-3}\right)$ lasting for $\sim 0.2$ years. Consequently, they have found production of s-process elements during the core He-flash of this star. On the other hand, Fujimoto et al. (2000) and Suda \& Fujimoto (2010) claim that no s-process signature would be present in the photosphere of low-mass stars that suffered the PIE during the core He-flash. A possible explanation for the difference between these studies is the shorter duration of the PIE in the models of Suda \& Fujimoto (2010). One-dimensional stellar evolution calculations of the PIE by all authors show that the convective He-burning region splits into a lower He-burning convective zone and an upper H-burning region (that later merges with the stellar envelope) after enough protons have been ingested into the He-burning region. However, conditions in the H-burning layers are not favorable for the s-process to happen. Because the convective zone in the Suda \& Fujimoto (2010) models splits before the s-process can take place, no s-process surface enrichment is observed. The current results in the literature for the PIE during the core He-flash are inconclusive with respect to the role that low-mass EMP stars play in the s-process production in the early Universe. Although the PIE is a well-established phenomenon in current 1D stellar evolution modeling, different studies do not agree about its main properties, and this is reflected in the production of s-process elements.

The aim of the present work is to explore the s-process production by low-mass EMP stars and their role as a source of s-process enhancement as observed in EMP stars. We investigate the main properties affecting the s-process production in these stars. We present evolutionary and s-process calculations for the core He-flash of $1 M_{\odot}$ stellar models. Our approach differs from Campbell et al. (2010) in that we use a more complete network that includes a neutron sink in the evolutionary calculations and a post-processing code for a posteriori calculations of s-process nucleosynthesis. We have also been able to follow the evolution of our models through the entire PIE and subsequent dredge-up phase. This has allowed us to directly obtain the surface enrichment in the models. In contrast, Campbell et al. (2010) estimated the surface enrichment from previous simulations (using a smaller network) due to numerical problems in following the subsequent dredge-up. We hope to close the gaps left by Campbell et al. (2010) by presenting models that include the evolution of the star during the dredge-up episode. In this way the necessity of extrapolating the resulting envelope abundances for all elements is prevented.

The paper is organized as follows: in Sects. 2 and 3, we describe the input physics of the codes used and the models calculated, respectively. We discuss the main characteristics of the PIE in Sect. 4 and the subsequent neutron and associated s-process production during the core He-flash in Sect. 5. We compare our results to the observations in Sect. 6.

\section{Stellar evolution and nucleosynthesis code}

In the calculations presented here, we have used the GARching STellar Evolution Code, GARSTEC, as described in Weiss \& Schlattl (2008). Convection is modeled using the mixing length theory (MLT) and the mixing length parameter $\alpha=1.75$, which is similar to the one obtained from standard solar model calculations. Mass loss is included using Reimers (1975) model with $\eta=0.4$. The OPAL equation of state (Rogers et al. 1996) was used. Radiative opacity tables are also from OPAL Iglesias \& Rogers (1996). At low temperatures, these data are complemented by those from Ferguson et al. (2005) and extended to account for carbon-rich compositions (see Weiss \& Ferguson 2009, for details). We have used the results by Itoh et al. (1983) for electron conduction opacities. For the present work, the nuclear network in GARSTEC has been extended. Changes are described in detail in the next section.

\subsection{Code implementation}

The version of GARSTEC from Weiss \& Schlattl (2008) follows the evolution of stable isotopes involved in the $\mathrm{p}-\mathrm{p}$ chain, the $\mathrm{CNO}$ cycles, and the standard helium burning reactions. For this work, the network in GARSTEC has been extended to include all relevant nucleosynthesis processes for intermediate mass elements $(A<30)$ during H-burning. This is important, because it has been suggested that elements in this mass range can be produced in metal-free primordial during H-burning (e.g., by $\mathrm{NeNa}$ and $\mathrm{MgAl}$ chains) and then act as seeds for the s-process (Goriely \& Siess 2001). Also, reactions that govern the production of neutrons during the He-burning have been incorporated in the network. In total, the network comprises of 34 isotopes linked by 120 reactions, including proton, alpha, and neutron captures, and beta decays.

For charged-particle reactions, the rates are from the NACRE database (Angulo et al. 1999) and the JINA REACLIB library (Cyburt et al. 2010). The exceptions are:

$$
\begin{aligned}
& -{ }^{14} \mathrm{~N}(\mathrm{p}, \gamma){ }^{15} \mathrm{O}-\text { Adelberger et al. (2011); } \\
& \text { - }{ }^{17} \mathrm{O}(\mathrm{p}, \gamma){ }^{18} \mathrm{~F} \text { and }{ }^{17} \mathrm{O}(\mathrm{p}, \alpha)^{14} \mathrm{~N}-\text { Moazen et al. (2007); } \\
& \text { - }{ }^{22} \mathrm{Ne}(\mathrm{p}, \gamma)^{23} \mathrm{Na}-\text { Hale et al. }(2002) ; \\
& \text { - }{ }^{23} \mathrm{Na}(\mathrm{p}, \gamma)^{24} \mathrm{Mg} \text { and }{ }^{23} \mathrm{Na}(\mathrm{p}, \alpha)^{20} \mathrm{Ne} \text { - Hale et al. (2004); } \\
& \text { - }{ }^{13} \mathrm{C}(\alpha, \mathrm{n})^{16} \mathrm{O} \text { - Kubono et al. (2003); }
\end{aligned}
$$


- ${ }^{22} \mathrm{Ne}(\alpha, \mathrm{n})^{25} \mathrm{Mg}$ - Jaeger et al. (2001); and

- ${ }^{12} \mathrm{C}(\alpha, \gamma){ }^{16} \mathrm{O}-$ Kunz et al. (2002).

Experimental rates for neutron capture reactions are taken from the recommended values given in the KADONIS database ${ }^{1}$ (Dillmann et al. 2006). For isotopes for which experimental data is not available, rates are taken from the JINA REACLIB ${ }^{2}$.

To accurately compute the abundance of neutrons during the evolutionary calculations so that it can be used for postprocessing calculations, the effect of neutron captures on all isotopes not included in the network has to be accounted for by using a neutron sink, which we call ${ }^{30} A A$, as described in Jorissen $\&$ Arnould (1989). The number fraction and the neutron capture cross-section of the sink element are given by

$$
\begin{aligned}
& Y_{A A}=\sum_{i={ }^{30} \mathrm{Si}}^{211} Y_{i} \\
& \sigma_{(A A, n)}(\tau)=\sum_{i={ }^{30} \mathrm{Si}} \frac{Y_{i}(\tau) \sigma_{(i, n)}}{Y_{A A}},
\end{aligned}
$$

respectively, where the summation extends over all the relevant isotopes that are not included in the evolutionary calculations. The sink cross-section $\left(\sigma_{A A, n}(\tau)\right)$ depends on the neutron capture path which is represented in this equation by the neutron exposure. The neutron exposure $\tau$ is given by $\tau(t)=\int_{0}^{t} n_{\mathrm{n}}\left(t^{\prime}\right) v_{T} \mathrm{~d} t^{\prime}$, where $n_{\mathrm{n}}$ is the neutron number density and $v_{T}$ is the thermal velocity of neutrons at temperature $T$.

One of the difficulties in the neutron sink approach is the calculation of the sink cross-section $\sigma_{(A A, n)}(\tau)$, which has to be known beforehand to get accurate estimates of the number of neutrons captured by heavy elements in the evolutionary calculations. This cross-section, however, depends on the distribution of heavy elements, and therefore on the neutron capture path. Thus, detailed s-process calculations are necessary to estimate values for $\sigma_{(A A, n)}(\tau)$.

In $A G B$ stars with a metallicity of $[\mathrm{Fe} / \mathrm{H}]>-2.5$, the s-process happens in a radiative environment, and the typical neutron exposure is smaller than $1.0 \mathrm{mb}^{-1}$. In the solar metallicity case, Herwig et al. (2003) have shown that the use of a fixed value for the sink cross-section in the calculations with the sink treatment leads to time evolution of the neutron exposure that is very similar to the results of calculations which include all isotopes up to $\mathrm{Pb}$. In their calculations, they found that a sink crosssection $\sigma_{(A A, n)}$ of $120 \mathrm{mb}$ could reproduce the neutron exposure evolution from the full s-process calculations within $10 \%$.

Conditions in EMP stars during the PIE are, however, different from those during the canonical thermal pulses in AGB stars. In the PIE neutrons are produced and consumed in convective regions. The large ingestion of protons that occurs in EMP stellar models either during the core He-flash for low-mass stars or early in the AGB for intermediate-mass stars leads to the production of a significant amount of ${ }^{13} \mathrm{C}$ through the reaction ${ }^{12} \mathrm{C}(\mathrm{p}, \gamma){ }^{13} \mathrm{~N}\left(\beta^{-}\right){ }^{13} \mathrm{C}$ and might result in neutron exposures that are much larger than those achieved in more metal-rich stars. To compute $\sigma_{(A A, n)}(\tau)$ as a function of the neutron irradiation $\tau$ (Eq. (2)) and to test the accuracy of the sink approach during the PIE in reproducing the time evolution of the neutron exposure, we therefore have performed a number of parametrized s-process calculations that we describe below.

\footnotetext{
http://www. kadonis.org/

2 https://groups.nscl.msu.edu/jina/reaclib/db/
}

For the parametrized s-process calculations, we take constant temperature and density values typically found during the PIE. We have performed a number of calculations covering the ranges of $T=1.0-2.5 \times 10^{8} \mathrm{~K}$ and $\rho=100-5000 \mathrm{~g} \mathrm{~cm}^{-3}$. In order to compute $\sigma_{(A A, n)}(\tau)$, we first perform network integrations using a full s-process network with 613 isotopes, which includes neutrons to ${ }^{211} \mathrm{Po}$. This network includes proton, neutron and alpha captures for all isotopes with $A<30$, and neutron captures for those with $A \geq 30$. In all cases, abundances of isotopes with $A<30$ are taken from a stellar model at the moment, where the PIE takes place. For isotopes with $A \geq 30$, we take scaled solar abundances (Grevesse \& Sauval 1998). The only exception is ${ }^{13} \mathrm{C}$, for which we took two different mass fraction values: $10^{-2}$ and $10^{-4}$. The outcome of these calculations is the time evolution of the abundances of all isotopes, which allow a direct evaluation of $\sigma_{(A A, n)}$ as a function of time (or neutron exposure; Eq. (2)). As a second step, we repeat the calculations but use the smaller network employed in GARSTEC for the evolutionary models and use the cross section derived in the first step for the neutron sink. The comparison of results of the neutron exposure in the first and second steps allows us to check the accuracy with which this quantity can be reproduced in simplified networks that use a neutron sink element.

Results are illustrated in Fig. 1, where we show the time evolution of the neutron exposure for the case of $T=10^{8} \mathrm{~K}$, $\rho=500 \mathrm{~g} \mathrm{~cm}^{-3}$, and the two choices of ${ }^{13} \mathrm{C}$ abundance. The neutron exposure derived using the full s-process network is shown as a black solid line. Results using the small network and the neutron sink with the capture cross-section, given by Eq. (2), are shown in dashed cyan line. The bottom panels show relative differences. Clearly, the sink approach performs very well and reproduces the neutron exposure of the full network calculation to a $0.1 \%$ accuracy. Additionally, we performed a test to check the sensitivity of neutron exposure predictions to the specific choice of $\sigma_{(A A, n)}$. For this exercise, we adopt the very simplistic approach of fixing $\sigma_{(A A, n)}=80 \mathrm{mb}$, i.e. to a constant value. Results are shown in dash-dotted red line. Even in this case, the neutron exposure is accurately reproduced to about $0.3 \%$. These results are representative of all parametrized calculations with different $T$ and $\rho$ values.

These results are much better than those found in the less extreme conditions present in AGB stars, as mentioned in previous paragraphs. The reason is simple. When protons are ingested into the helium-burning region during the PIE, light metals have already been produced. These primary metals, predominantly ${ }^{12} \mathrm{C}$, amount to a mass fraction of about 0.05 , and they are about $10^{8}$ times more abundant than metals with $A \geq 30$. The PIE only takes place in EMP models. Taking a typical average neutron cross-section for the light and heavy elements of $0.01 \mathrm{mb}$ and $1000 \mathrm{mb}$, respectively, the average contribution to the neutron capture by light elements is much larger than by heavy elements in this environment. We estimate $\sigma_{\text {light }} \mathrm{X}_{\text {light }} / \sigma_{\text {heavy }} \mathrm{X}_{\text {heavy }} \sim 1500$. Almost all neutrons are captured by elements with $A<30$ in the environment associated with the PIE, and these capture reactions are always treated in detail in our models. Therefore, the value of $\sigma_{(A A, n)}$ used during a PIE is of little consequence for predicting a neutron exposure consistent with that derived from full network calculations.

In this work, all post-processing calculations are based upon evolutionary calculations, where the neutron capture crosssection of the sink is given as a function of the neutron exposure, as derived from Eq. (2). For each parametrized calculation, a fitting formula for $\sigma_{(A A, n)}$ as a function of $\tau$ is derived. These formulae are then used in the evolutionary calculations to compute 

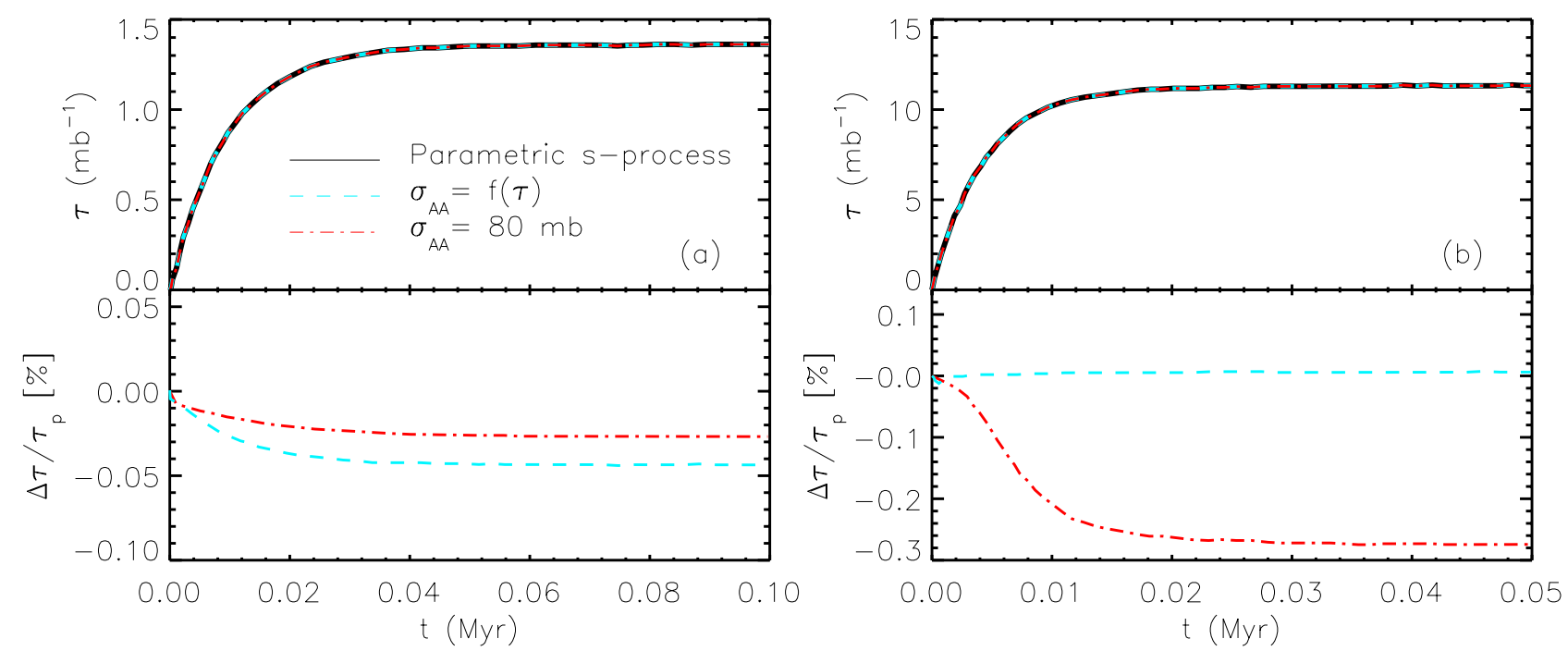

Fig. 1. Top panels: time evolution of the neutron exposure for the parametrized s-process calculation and the neutron sink approach with different cross-section values for a composition of heavy elements $(A>30)$, corresponding to a solar abundance pattern scaled down to $Z=10^{-8}$. The abundance values for light elements $(A<30)$ were taken from stellar models calculated using GARSTEC except for ${ }^{13} \mathrm{C}$, where we take two different values. Panel a) shows results for a ${ }^{13} \mathrm{C}$ mass-fraction of $10^{-4}$ and panel b) for a ${ }^{13} \mathrm{C}$ mass-fraction of $10^{-2}$. In both cases, lines depicting results for the full network (black line) and a sink element (cyan and red lines) overlap. To enhance differences for each value of the ${ }^{13} \mathrm{C}$ abundance, we show the relative difference in percentage of the neutron exposure with respect to the neutron exposure from the parametric calculations $\left(\tau_{\mathrm{p}}\right)$ overlapped in both plots.

the neutron capture cross-section of the sink element. As just discussed, the detailed choice of this quantity is of very little consequence for the s-process calculations.

\subsection{Post-processing program}

The post-processing code uses the model structure (temperature, density, neutron abundance, and convection velocity) from evolutionary calculations as input for the s-process calculations. The integration of the nuclear network is done between two structural models, and the evolutionary timestep between them is divided into smaller burning and mixing steps. All structural quantities are kept constant between the two stellar models. Within each substep, we first compute changes in chemical composition due to nuclear burning and then mix the convective (and overshooting) regions.

We adopted the time-dependent mixing scheme described by Chieffi et al. (2001):

$X_{i}^{k}={ }^{0} X_{i}^{k}+\frac{1}{M_{\text {mixed }}} \sum_{j=\text { mixed }}\left({ }^{0} X_{j}^{k}-{ }^{0} X_{i}^{k}\right) f_{i j} \Delta M_{j}$,

where the sum extends over the mixed region (including overshooting regions if present); $\Delta M_{j}$ is the mass of the shell $j ;{ }^{0} X_{i}^{k}$ and ${ }^{0} X_{j}^{k}$ are, respectively, the abundances of isotope $k$ in the shells $i$ and $j$ before mixing; $M_{\text {mixed }}$ is the mass of the entire mixed region; and $f_{i j}$ is a damping factor given by

$f_{i j}=\min \left(\frac{\Delta t}{\tau_{i j}}, 1\right)$

that accounts for partial mixing between zones $i$ and $j$, when the mixing timescale $\tau_{i j}$ between them is longer than the timestep used in the calculations, $\Delta t$. Here, $\tau_{i j}$ is assumed to be the convective turn-over timescale between grid points $i$ and $j$, and the convective velocity is determined using MLT. With this scheme, we account for partial mixing occurring when the convective turn-over timescale between two shells in the model is longer than the mixing timestep. This scheme is very easily implemented and, importantly, much faster than the diffusive approach used in GARSTEC. With a test version of the postprocessing code where the mixing is treated by solving the diffusion equations, we have checked that both schemes agree very well for sufficiently small timesteps.

Weak interaction rates (electron captures and $\beta$ decays) are taken mainly from Takahashi \& Yokoi (1987) and interpolated as a function of temperature and electron density. At temperatures lower than $10^{7} \mathrm{~K}$, we have assumed a constant value equal to the laboratory rate. Sources for the neutron capture rates are the same used for the network in GARSTEC, which are either from the JINA REACLIB library or calculated using the newest KADONIS results. Proton and alpha captures are not included in the network, since only isotopes heavier than ${ }^{29} \mathrm{Si}$ are included in the post-processing, and the relevant reactions for them are the neutron captures and beta decays.

The network includes 580 isotopes (starting from ${ }^{30} \mathrm{Si}$ ) and more than 1000 reactions. Branching points involving the most well-known isomers are included and the network terminates with the $\alpha$ decays of Po isotopes. All isotopes with decay lifetimes comparable to or longer than the lifetime against neutron captures have been included in the network.

\section{Models}

We performed evolutionary calculations of $1 M_{\odot}$ stellar models from the zero-age main sequence to the dredge-up after the PIE. The composition is solar scaled with metallicities $Z=0$ and $Z=10^{-8}$. Our standard models (M1 and M2 - see Table 1) do not include overshooting or metal diffusion.

Mixing in convective regions is modeled as a diffusive process. The diffusion coefficient in convective regions is taken as $D_{\mathrm{c}}=1 / 3 v \times \ell$, where $v$ is the convective velocity derived from MLT and $\ell$ is the mixing length. In the PIE, it has been suggested that, the reactive nature of the hydrogen material adds some buoyancy to the sinking elements and this results in a reduced efficiency of the mixing (Herwig et al. 2011). We have 
Table 1. Main properties of proton ingestion episode.

\begin{tabular}{lcccccc}
\hline \hline Model & $\begin{array}{c}\log L_{\max }^{\mathrm{He} a} \\
{\left[L_{\odot}\right]}\end{array}$ & $\begin{array}{c}\log L_{\max }^{\mathrm{H} b} \\
{\left[L_{\odot}\right]}\end{array}$ & $\begin{array}{c}M_{\max }^{\mathrm{He} c} \\
{\left[M_{\odot}\right]}\end{array}$ & $\begin{array}{c}M_{c}^{\mathrm{He} d} \\
{\left[M_{\odot}\right]}\end{array}$ & $\begin{array}{c}\Delta t_{\operatorname{mix}}{ }^{e} \\
{[\mathrm{yr}]}\end{array}$ & $\begin{array}{c}\Delta t_{\mathrm{PIE}}{ }^{f} \\
{[\mathrm{yr}]}\end{array}$ \\
\hline $\mathrm{M}^{g}$ & 9.972 & 10.48 & 0.171 & 0.475 & 1.400 & 1.356 \\
$\mathrm{M} 2$ & 10.70 & 10.71 & 0.237 & 0.508 & 0.020 & 0.432 \\
$\mathrm{M}^{h}$ & 10.69 & 9.736 & 0.236 & 0.508 & 0.027 & 0.482 \\
$\mathrm{M}^{i}$ & 10.70 & 11.01 & 0.237 & 0.509 & 0.018 & 0.192 \\
$\mathrm{M}^{j}$ & 10.69 & 11.03 & 0.237 & 0.509 & $7 \times 10^{-3}$ & 0.043 \\
$\mathrm{Z}^{k}$ & 11.10 & 10.37 & 0.267 & 0.529 & 0.200 & 2.204 \\
\hline
\end{tabular}

Notes. ${ }^{(a)}$ Logarithm of the maximum He-burning. ${ }^{(b)}$ Logarithm of the maximum H-burning. ${ }^{(c)}$ Position of maximum energy released by He-burning. ${ }^{(d)}$ Degenerate core mass. ${ }^{(e)}$ Time between maximum Heburning luminosity and the onset of hydrogen mixing. ${ }^{(f)}$ Time between the onset of hydrogen mixing and the splitting of the convective zone. (g) Model with $Z=0$. All other models, except $Z 7$, have $Z=10^{-8}$. (h) Model with convective efficiency reduced by an order of magnitude. ${ }^{(i)}$ Model that includes overshooting with $f=0.016 .{ }^{(j)}$ Model that includes overshooting with $f=0.07$. ${ }^{(k)}$ Model with initial $Z=10^{-7}$.

mimicked this situation by using a reduced diffusion coefficient by dividing the above relation by 10 in a model with $Z=10^{-8}$ (model M3 - Table 1).

We have also computed additional models with metallicity $Z=10^{-8}$ which include overshooting. Overshooting has been modeled as an exponentially decaying diffusive coefficient, as described in Freytag et al. (1996) and has been included in all convective boundaries. To test the influence and associated uncertainties of this poorly-understood phenomenon, two values have been chosen of the free parameter $f$ that determines the extension of the overshooting regions: $f=0.016$ and 0.07 (models M4 and M5 respectively - Table 1). The first value is a standard choice for this prescription of overshooting, because its results in the main sequence are comparable to the canonical choice of 0.2 pressure scale heights (Magic et al. 2010), a value known to reproduce different observational constraints such as the width of the main sequence observed in different open clusters. The higher value is intended to represent a case where overshooting is much larger than in convective cores and deep stellar envelopes. This is closer to what was originally found by Freytag et al. (1996) for thin convective envelopes of A-type stars. Incidentally, this amount of overshooting gives a lithium depletion in solar models (Schlattl \& Weiss 1999) that is consistent with observations.

Finally, we have computed an additional model, Z7, with the same physical inputs as model M2 but with an initial metallicity $Z=10^{-7}$, which is one order of magnitude larger. This allows us to consider the effect of the initial metallicity on the production of elements and to compare to HE0107-5240.

\section{Main characteristics of the proton ingestion episode}

In the following sections, we use model M2 (See Table 1) to illustrate the characteristics of the PIE, unless stated otherwise.

Low-mass stars ignite He-burning under degenerate conditions, leading to the well known core He-flash. Due to stronger neutrino cooling in the innermost core, the ignition point is offcenter, as shown by the position of the maximum energy release by He-burning $M_{\max }^{\mathrm{He}}=0.237 M_{\odot}$ (Fig. 2). Ignition of He-burning results in the formation of the so-called helium convective zone $(\mathrm{HeCZ})$, the outer boundary of which advances in mass during the development of the He-flash. In Fig. 2, we
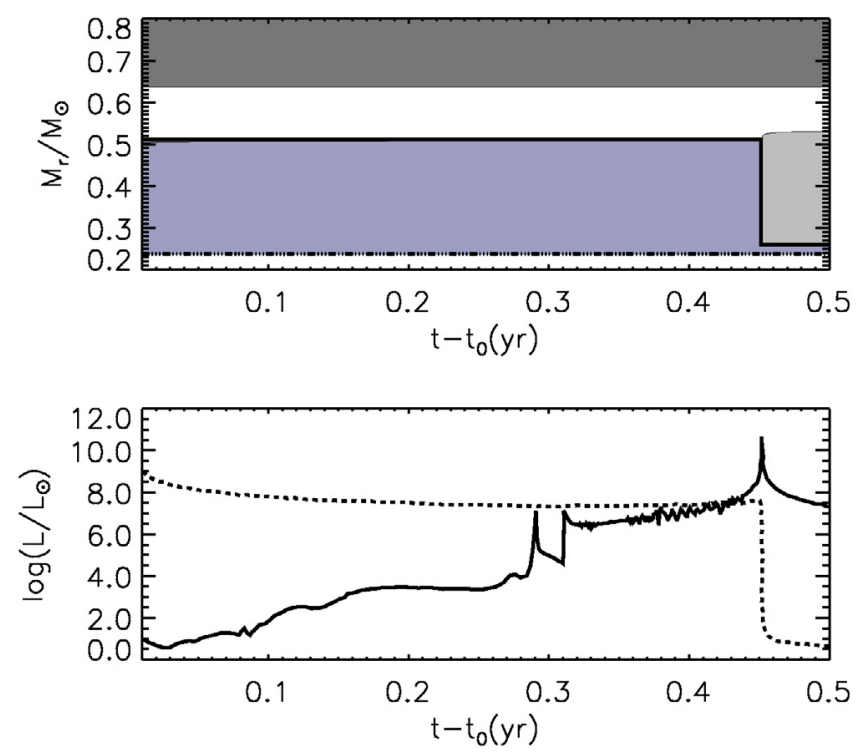

Fig. 2. Upper panel: time evolution of convective zones in model M2 $Z=10^{-8}$ during the PIE. Dark grey area: convective envelope, purple area between 0.24 and $0.51 M_{\odot}$ : HeCZ; light grey area: HCZ. Thick solid line represents the position where hydrogen mass fraction is 0.001 ; dot-dashed line represents the position of maximum energy release due to He-burning, respectively. The splitting of the $\mathrm{HeCZ}$ around $t-t_{0}=$ $0.451 \mathrm{yr}$ is evident with the formation of the detached HCZ. Bottom panel: evolution of H-burning (solid line) and He-burning (dashed line) luminosities. $t=t_{0}$ corresponds to the time of maximum He-burning luminosity.

show the evolution of the $\mathrm{HeCZ}$ (purple region extending from $M_{r} / M_{\odot}=0.237$ up to 0.510 ) during the time following the maximum helium-burning luminosity up to the moment where the PIE starts. The combination of an off-center He-burning ignition and a low entropy barrier between the He- and H-burning shells in the very metal-poor environment allows the $\mathrm{HeCZ}$ to reach hydrogen-rich layers (Hollowell et al. 1990), giving rise to the PIE.

The $\mathrm{HeCZ}$ reaches the radiatively stratified $\mathrm{H}$-rich layers on top (initially represented by a white region above $M_{r} / M_{\odot}=$ 0.510 ) soon after the He-burning luminosity reaches its maximum value. Protons are then mixed down into the $\mathrm{HeCZ}$ and are captured by the abundant ${ }^{12} \mathrm{C}$, creating ${ }^{13} \mathrm{C}$. The H-burning luminosity increases, while the $\mathrm{HeCZ}$ continues expanding (in mass). Note the penetration of the $\mathrm{HeCZ}$ into the H-rich region occurs in a narrow region of only $\sim 10^{-3} M_{\odot}$, and it is barely visible around $0.510 M_{\odot}$ in Fig. 2 . Around 0.451 years after the He-luminosity reached its maximum, a secondary flash happens, the $\mathrm{H}$-flash, with a peak H-burning luminosity comparable to the He-burning luminosity $\left(\log L_{\max }^{\mathrm{He}} / L_{\odot}=10.70\right.$, $\left.\log L_{\max }^{\mathrm{H}} / L_{\odot}=10.71\right)$. The burning timescale for hydrogen through the ${ }^{12} \mathrm{C}(\mathrm{p}, \gamma)$ reaction becomes shorter as protons travel downwards in the $\mathrm{HeCZ}$ until a point inside the $\mathrm{HeCZ}$ is reached where the burning and mixing timescales are comparable. At this position $\left(M_{\max }^{\mathrm{H}}=0.259 M_{\odot}\right)$, the energy release by H-burning is at a maximum and the temperature rises quickly, resulting in an inversion in the temperature profile that eventually leads to the splitting of the convective zone. This prevents the penetration of protons further down into the $\mathrm{HeCZ}$.

The splitting results in two convective zones: a small $\mathrm{HeCZ}$ and an upper convective zone, which is referred to as hydrogen convective zone (HCZ). The outer boundary of the HCZ continues to advance in mass. In about 1100 years, the convective envelope deepens and products of burning during and after the PIE 
Table 2. Surface abundances.

\begin{tabular}{lccccc}
\hline \hline Element $^{a}$ & $M 2$ & $M 3$ & $M 4$ & $M 5$ & $Z 7$ \\
\hline $\mathrm{C}$ & 6.97 & 6.80 & 6.99 & 6.90 & 6.25 \\
$\mathrm{~N}$ & 7.05 & 6.73 & 7.04 & 7.20 & 5.91 \\
$\mathrm{O}$ & 5.64 & 5.46 & 5.69 & 5.81 & 4.83 \\
$\mathrm{Sr}$ & 1.49 & 1.59 & 1.86 & 2.78 & 1.88 \\
$\mathrm{Y}$ & 1.86 & 1.91 & 2.04 & 2.59 & 2.23 \\
$\mathrm{Zr}$ & 1.92 & 2.05 & 2.21 & 3.17 & 2.33 \\
$\mathrm{Ba}$ & 3.16 & 2.77 & 3.06 & 3.96 & 3.13 \\
$\mathrm{La}$ & 3.27 & 2.76 & 3.37 & 4.29 & 3.24 \\
$\mathrm{Eu}$ & 2.97 & 2.32 & 3.04 & 3.89 & 2.81 \\
$\mathrm{~Pb}$ & 4.74 & 4.86 & 5.00 & 4.52 & 5.31 \\
\hline
\end{tabular}

Notes. ${ }^{(a)}$ All abundances are given in terms of: $[\mathrm{X} / \mathrm{Fe}]=\log _{10}(\mathrm{X} / \mathrm{Fe})_{\star}-$ $\log _{10}(\mathrm{X} / \mathrm{Fe})_{\odot}$.

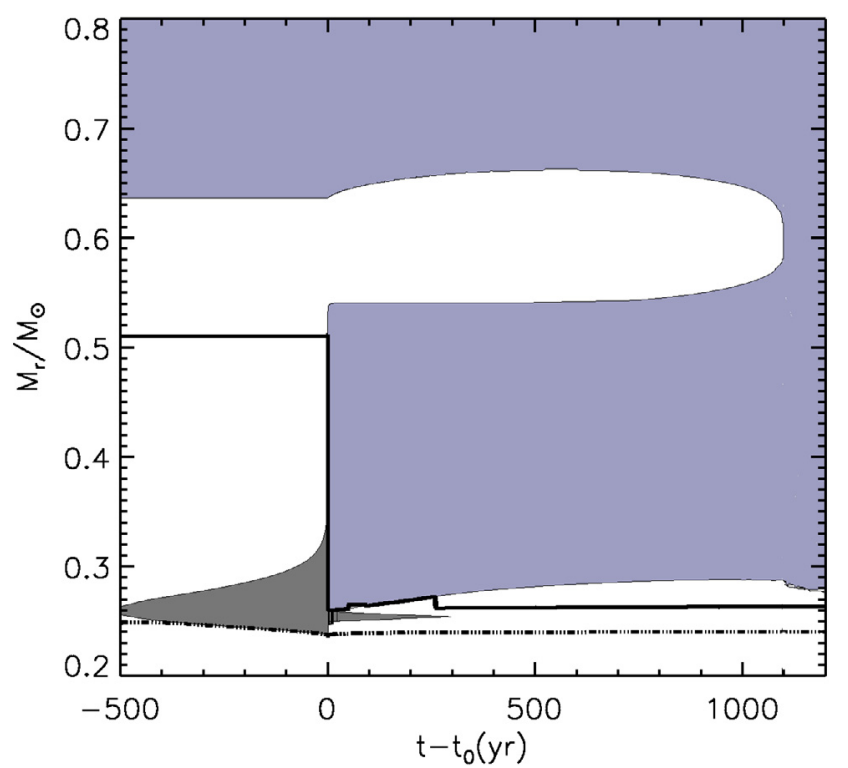

Fig. 3. Long-term evolution of the $1 M_{\odot}, Z=10^{-8}$ stellar model during the development of the core He-flash, the PIE, and the subsequent dredge-up event. $t=t_{0}$ corresponds to the time of maximum $\mathrm{He}-$ burning luminosity. As in Fig. 2, thick solid and dot-dashed lines represent the position of maximum energy release due to H-burning and He-burning, respectively. Merging of the HCZ and the stellar envelope occurs around $1100 \mathrm{yr}$ after the PIE takes place.

are dredged-up to the surface. The envelope is enriched mainly in $\mathrm{C}, \mathrm{N}$, and $\mathrm{O}$. Similarly to results by other authors, the amount of $\mathrm{C}$ and $\mathrm{N}$ brought up to the surface is too large compared to the abundances observed in EMP stars (values in Table 2 are more than 3 dex larger than the average CEMP's carbon abundance). Enrichment in s-process depends strongly on the nucleosynthesis before the splitting of the $\mathrm{HeCZ}$ and is discussed in the following section. The long-term evolution before and after the PIE is illustrated in Fig. 3, where time has been set to zero at the moment when $L^{\mathrm{He}}$ reaches its maximum.

The time between maximum He-burning luminosity and the onset of hydrogen mixing $\left(\Delta t_{\text {mix }}\right)$ in our calculations is usually larger than the interval found in other studies (Hollowell et al. 1990; Suda et al. 2007; Picardi et al. 2004). This might partially be the result of the larger mass between the bottom of the HeCZ and the location of base of the H-rich layer in our models. Our predicted $\Delta t_{\text {mix }}$ varies from $10^{-2}$ to 1 year (depending on the metallicity and the convection efficiency assumed in the models), while this value ranges from $10^{-3}$ to $10^{-2}$ years in other studies. The main properties of the core helium flash and the PIE are summarized in Table 1 . An important property of the PIE for the s-process is the time between the onset of hydrogen mixing and the splitting of the convective zone, which we refer to here as $\Delta t_{\text {PIE }}$. After the splitting of the $\mathrm{HeCZ}$, the abundance of ${ }^{14} \mathrm{~N}$, the main neutron poison in this environment, in the HCZ rapidly builds up. As the ${ }^{13} \mathrm{C}$ to ${ }^{14} \mathrm{~N}$ ratio drops below unity, neutrons are predominantly captured by ${ }^{14} \mathrm{~N}(\mathrm{n}, \mathrm{p}){ }^{14} \mathrm{C}$ and the s- process is effectively shut down. Therefore, $\Delta t_{\mathrm{PIE}}$ determines the timescale for s-processing. In our calculations, $\Delta t_{\mathrm{PIE}}$ is in the range $\sim 0.1$ to $1 \mathrm{yr}$ (Table 1), which is about an order of magnitude longer than in some other studies (e.g. Hollowell et al. 1990). In contrast, Campbell et al. (2010) presented a model with a metallicity of $[\mathrm{Fe} / \mathrm{H}]=-6.5$ where $\Delta t_{\mathrm{PIE}} \sim 20 \mathrm{yrs}$, which is about a factor of 50 larger than our findings. According to our calculations, differences in $\Delta t_{\text {PIE }}$ imply differences in the production of $\mathrm{s}$-process elements. Therefore, the large variations found in $\Delta t_{\mathrm{PIE}}$ as described, which span up to three orders of magnitude, are probably the reason why results for nucleosynthesis of s-process elements from different authors range from no production at all, as seen for example in Suda \& Fujimoto (2010), to extremely large production factors, as seen in Campbell et al. (2010), and described in Sect. 1.

We note here that splitting of the $\mathrm{HeCZ}$ is ubiquitous in all 1-dimensional simulations of the PIE during the core helium flash (Hollowell et al. 1990; Schlattl et al. 2001; Campbell \& Lattanzio 2008). Splitting of the HeCZ (shell) is also found in PIE resulting from very late thermal pulses in post-AGB stars (Herwig 2001; Althaus et al. 2005) and in simulations of the early phases of the TP-AGB in very metal-poor stars (Fujimoto et al. 2000; Serenelli 2006; Cristallo et al. 2009). On the other hand, recent hydrodynamic 3-dimensional simulations of the TP-AGB phase for a low-mass star (Stancliffe et al. 2011) have not shown splitting of the $\mathrm{HeCZ}$, although the authors argued this might be an artifact of the low resolution used in the calculations.

\section{Neutron production and the s-process}

During the PIE, ${ }^{13} \mathrm{C}$ is formed by proton captures on ${ }^{12} \mathrm{C}$ and mixed throughout the entire $\mathrm{HeCZ}$. The abundance of ${ }^{13} \mathrm{C}$ in the convective zone increases by several orders of magnitude during $\Delta t_{\mathrm{PIE}}$ (from $X_{13 \mathrm{C}} \sim 10^{-10}$ to $X_{13 \mathrm{C}} \sim 10^{-3}$ in mass-fraction).

Neutrons are produced by the reaction ${ }^{13} \mathrm{C}(\alpha, \mathrm{n}){ }^{16} \mathrm{O}$, near the bottom of the convective zone, reaching maximum neutron density values larger than $10^{14} \mathrm{~cm}^{-3}$. Besides ${ }^{4} \mathrm{He},{ }^{12} \mathrm{C}$ is the most abundant isotope in the $\mathrm{HeCZ}$, and thus neutrons are mainly captured by it and enter into the recycling reaction ${ }^{12} \mathrm{C}(\mathrm{n}, \gamma){ }^{13} \mathrm{C}(\alpha, \mathrm{n}){ }^{16} \mathrm{O}$. At the beginning of the proton ingestion, ${ }^{13} \mathrm{C}$ is much less abundant than ${ }^{14} \mathrm{~N}$, and neutrons not captured by ${ }^{12} \mathrm{C}$ are captured by ${ }^{14} \mathrm{~N}$. As the PIE progresses, the ${ }^{13} \mathrm{C}$ abundance, the ${ }^{13} \mathrm{C} /{ }^{14} \mathrm{~N}$ abundance ratio, and the neutron density increase (Figs. 4 and 5). Eventually, the ${ }^{13} \mathrm{C} /{ }^{14} \mathrm{~N}$ abundance ratio gets larger than unity, and more neutrons are free to be captured by heavy elements. The ${ }^{13} \mathrm{C} /{ }^{14} \mathrm{~N}$ abundance ratio reaches a maximum value at around $\Delta t \sim 0.18$ yrs after the PIE starts. Hydrogen abundance is now large enough to enhance the competition between ${ }^{13} \mathrm{C}(\mathrm{p}, \gamma){ }^{14} \mathrm{~N}$ and ${ }^{13} \mathrm{C}(\alpha, \mathrm{n}){ }^{16} \mathrm{O}$. Thus, the ${ }^{13} \mathrm{C} /{ }^{14} \mathrm{~N}$ abundance ratio starts to decrease, leading to a shallower increase in neutron density than before. After the splitting, the bottom of the HeCZ moves slightly outwards and cools down. The supply of fresh protons into the HeCZ stops, and consequently, the neutron flux is quickly suppressed (Fig. 4). 
M. A. Cruz et al.: S-process in extremely metal-poor, low-mass stars
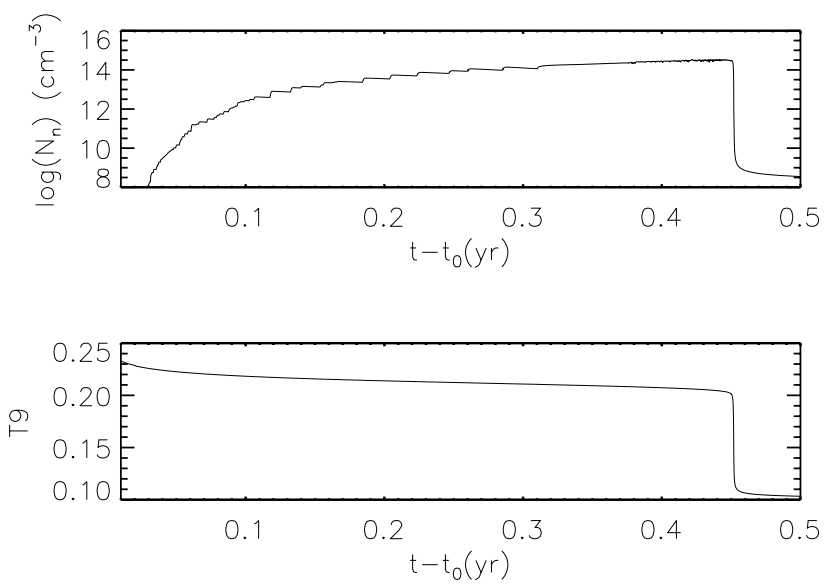

Fig. 4. Upper panel: time evolution of maximum neutron density in our $Z=10^{-8}$ model (M2) at $M=0.237 M_{\odot}$. Bottom panel: evolution of the temperature near the bottom of the HeCZ. In both cases, the sudden change at $t-t_{0} \approx 0.451 \mathrm{yr}$ reflects the splitting of the HeCZ.

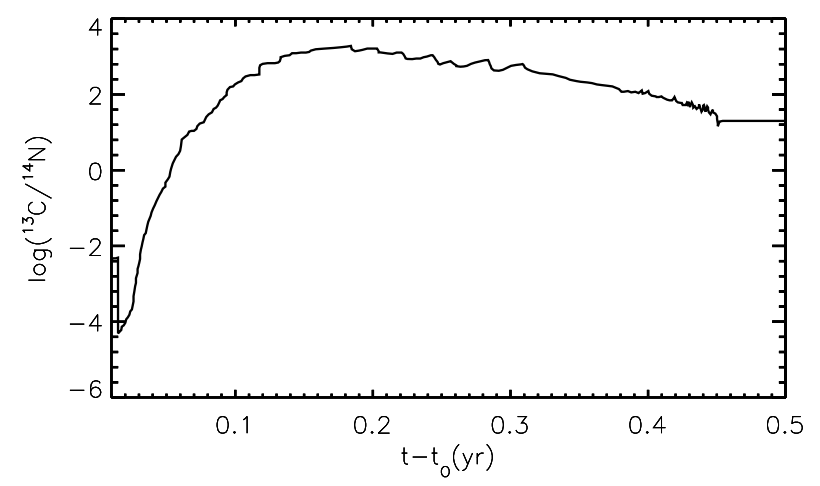

Fig. 5. ${ }^{13} \mathrm{C} /{ }^{14} \mathrm{~N}$ ratio near the bottom of the $\mathrm{HeCZ}$. The value of $t_{0}$ is the same as in Figs. 2 and 4.

Regarding the production of s-process elements in a given environment (which determines, among others, the number of seed nuclei present), the key quantity is the time-integrated neutron flux, or neutron exposure, given by

$\tau=\int v_{T} n_{\mathrm{n}} \mathrm{d} t$

where $v_{T}$ is the thermal velocity of neutrons and $n_{\mathrm{n}}$ their number density. In the case of the PIE, the other relevant quantity is the splitting timescale $\Delta t_{\mathrm{PIE}}$ which, as we discuss below, determines the timespan over which neutrons are effectively produced and therefore determines the cutoff to the integration in Eq. (5).

In Fig. 6, we show the neutron exposure as a function of time near the bottom of the HeCZ by solid lines, where the maximum neutron flux is achieved. Results for the reference model, M2, are depicted in red. In the same figure, dashed lines denote the neutron exposure averaged across the entire convective zone. For comparison, typical values for the neutron exposure during the AGB phase are roughly two orders of magnitude smaller than the maximum neutron exposure at the bottom of the HeCZ.

At the beginning of the PIE, iron-peak elements (mainly ${ }^{56} \mathrm{Fe}$ ) are converted to elements of the first s-process peak ( $\mathrm{Sr}, \mathrm{Y}$, $\mathrm{Zr})$. The ${ }^{56} \mathrm{Fe}$ abundance is strongly reduced, reaching its minimum value at about $0.27 \mathrm{yrs}$ after the maximum helium-burning luminosity. Between 0.27 and 0.32 yrs after the He-flash, second s-process peak elements are produced and are followed shortly after by the producion of the heaviest elements $(\mathrm{Pb}$ and $\mathrm{Bi})$.

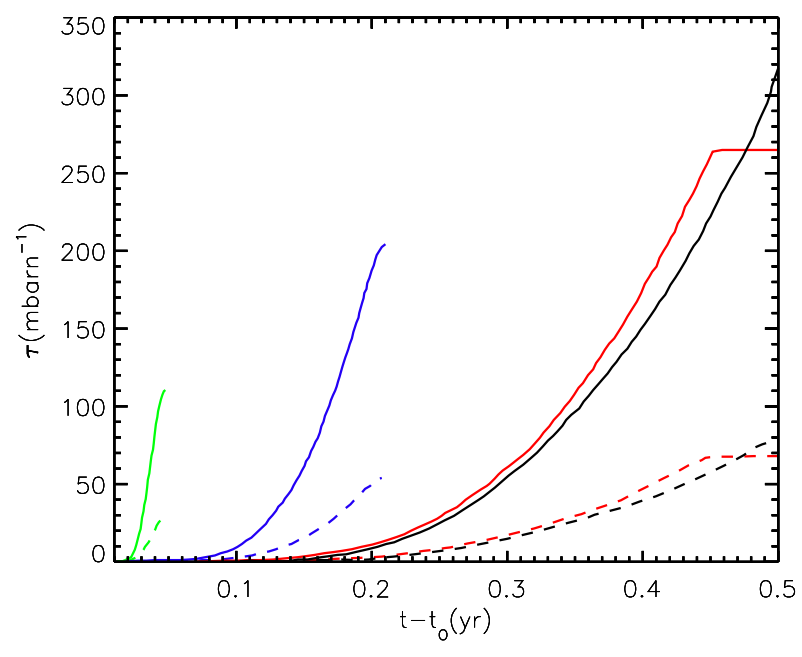

Fig. 6. Time evolution of neutron exposure. Solid lines represent the neutron exposure at the position of maximum neutron density (i.e., near the bottom of the $\mathrm{HeCZ}$ ) and dashed lines represent the average neutron exposure in the convective zone. Red line: standard $Z=10^{-8}$ model (M2); black line: reduced convection efficiency (M3); blue line: model including overshooting $(f=0.016-\mathrm{M} 4)$; and green line: model including overshooting $(f=0.07-$ M5).

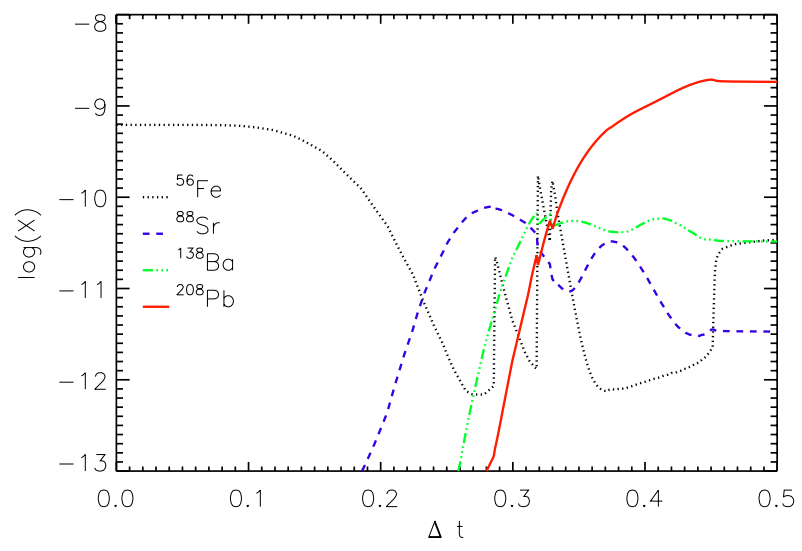

Fig. 7. Evolution of the mass fraction of isotopes representative of the three s-process abundance peaks and ${ }^{56} \mathrm{Fe}$ as a function of time. These abundances were sampled at the position $0.4 M_{\odot}$. They are representative of the entire convective zone and, after the splitting, are located in the $\mathrm{HCZ}$ which later comes into contact with the stellar envelope. The peaks, most noticeable for ${ }^{56} \mathrm{Fe}$, result from episodic advance of the outer convective boundary due to limitations in the time and spatial resolution.

The mass fraction of $\mathrm{Fe}, \mathrm{Sr}, \mathrm{Ba}$, and $\mathrm{Pb}$ in the convective zone are shown in Fig. 7. The spikes seen in the Fe abundance reflect episodic advances of the convective shell over the radiative upper layers, which dredge-down fresh material. Near the end of the PIE around $t-t_{0} \approx 0.45 \mathrm{yr}$, the outer boundary of the $\mathrm{HCZ}$ advances, where freshly dredged-down iron increases the ${ }^{56} \mathrm{Fe}$ abundance by an order of magnitude but the neutron density simultaneously drops several orders of magnitude as already shown in Fig. 4 and the s-process is quenched.

After the splitting of the $\mathrm{HeCZ}$, the upper convective zone, $\mathrm{HCZ}$, penetrates further into the $\mathrm{H}$-rich layers above, and ${ }^{12} \mathrm{C}$ is converted into ${ }^{14} \mathrm{~N}$. The cooling of the HCZ effectively switches off $\alpha$-captures by ${ }^{13} \mathrm{C}$, and ${ }^{14} \mathrm{~N}$ captures most of the remaining neutrons available, effectively quenching s-processing. Therefore, the abundances of heavy elements in the HCZ remain unchanged by nuclear burning after the splitting. They do not 
change until the HCZ merges with the stellar envelope, and the processed material is dredged-up to the surface.

\subsection{The influence of convective mixing}

The treatment of convection and the associated mixing in stellar evolution calculations is subject to fundamental uncertainties that cannot be properly addressed in 1D models. Nevertheless, we can try to understand how varying the mixing efficiency in convective regions or the extent of the mixing regions (e.g., by including overshooting) affect the basic properties of the PIE. In turn, this affects neutron production and the ensuing creation of s-process elements.

Let us first discuss models including overshooting. Model M4 is computed by using a moderate amount of overshooting characterized by $f=0.016$ (Sect. 3 ). The extended convective boundary in this model allows a larger penetration of the $\mathrm{HeCZ}$ into H-rich layers. In this model, the splitting of the $\mathrm{HeCZ}$ occurs at $M_{\max }^{\mathrm{H}}=0.244 M_{\odot}$, which is deeper than for model M2 for which $M_{\max }^{\mathrm{H}}=0.259 M_{\odot}$. More fuel is ingested into the $\mathrm{HeCZ}$ and thus a faster, but shorter, evolution of the PIE is achieved. Comparison of models M2 and M4 (red and blue lines, respectively, in Fig. 6) show that the more vigorous entrainment of hydrogen into the $\mathrm{HeCZ}$ in model M4 produces an early and steeper increase in the neutron exposure. Furthermore, the maximum neutron flux is almost a factor of two larger in model M4 than in model M2. However, the faster release of nuclear energy by hydrogen burning leads to the PIE lasting less than half the time it does in the case of no overshooting. This leads to a significantly smaller final neutron exposure in the case of M4 as seen in Fig. 6. With a large overshooting parameter $f=0.07$, model M5 confirms this trend. For this model, the results shown in green in Fig. 6 confirm that a larger overshooting region leads to a more violent hydrogen ingestion (as seen by the quick and steep rise in neutron production) but of shorter duration and an overall reduced efficiency in the final neutron exposure. Quantitatively, the difference between the final neutron exposure for the two models with overshooting is not too large, despite the $\sim 50 \%$ difference in $\Delta t_{\mathrm{PIE}}$ (see blue and green solid lines in Fig. 6).

As mentioned before, we have found shorter splitting timescales than other recent calculations (e.g. those of Campbell et al. 2010). To mimic these longer splitting timescales, we computed a model with a reduced convective mixing efficiency. In our calculations, we have simply achieved this by dividing the diffusion coefficient by a constant factor. In model M3, this reduction is by one order of magnitude. The slower mixing is reflected by the outer location of the splitting at $M_{\max }^{\mathrm{H}}=0.303 M_{\odot}$. As expected, a reduced mixing efficiency results in a larger $\Delta t_{\mathrm{PIE}}$, although the difference is not too significant compared to the standard case (see Models M2 and M3 in Table 1). This increase in time produces a total neutron exposure at a maximum (black solid line) of about $20 \%$ larger than in the standard case; this should favor the production of the heaviest s-process isotopes, as final $\mathrm{Pb}$ abundances given in Table 2 show.

In a radiative environment, the neutron exposure at the position of maximum neutron density would result in a strong enhancement in $\mathrm{Pb}$ due to the repeated neutron captures in a static environment in all cases. However, the interplay between burning and convection is important in a convective environment. The mixing timescale is shorter than the overall phase of s-process production and, thus, the relevant quantity for the final production is the averaged neutron exposure. Figure 6 shows the neutron exposure averaged over the convectively mixed zone (dashed lines). As it can be seen, the averaged neutron exposure is strongly reduced in the overshooting model (blue dashed line), but it is not much affected by the reduction in the mixing efficiency (black dashed line). This leads to small differences in the final production of s-process elements despite the $50 \%$ difference in the neutron exposure at the bottom of the HeCZ.

\subsection{The dependence on metallicity}

During the PIE, the amount of available neutrons do not depend, at least directly, on the initial metallicity of the star, because the necessary ${ }^{12} \mathrm{C}$ is produced by the helium-core flash. Similarly, the abudance of the most relevant neutron poison in this context, ${ }^{14} \mathrm{~N}$, does not depend on the initial metallicity. In contrast, the number of seed nuclei is a linear function of metallicity (assuming the distribution of relative abundances is not altered). However, the development of the PIE may be altered by the metallicity of the model. For model Z7, which has $Z=10^{-7}$, we have found a slightly more gentle PIE event, where $\log L_{\max }^{\mathrm{H}} / L_{\odot}=10.37$. This value is more than a factor of two lower than for model M2, which differs from model Z7 only in the initial $Z$ value adopted. However, $\Delta t_{\mathrm{PIE}}=2.2 \mathrm{yr}$, which is a factor of 5 longer, and the integrated neutron exposure at the point of maximum neutron density reaches a very large value of $370 \mathrm{mb}^{-1}$, while the averaged total neutron exposure is $100 \mathrm{mb}^{-1}$. These values are larger than for model M2. The production of s-process elements reflects the scenario depicted above, and it is roughly one order of magnitude larger as a result of the larger abundance of seed nuclei but almost the same abundance of poison elements when compared to the more metal poor model M2. We come back to this in Sect. 6.

\subsection{The absence of iron-peak seeds: zero metallicity case}

Goriely \& Siess (2001) have studied the occurrence of s-process nucleosynthesis during the AGB phase of a $3 M_{\odot}$ stellar model with initial zero metallicity. By parametrizing the formation of the ${ }^{13} \mathrm{C}$-pocket during the third dredge-up (TDU), they found that neutron densities of the order of $10^{9} \mathrm{~cm}^{-3}$ can be reached. Furthermore, their results showed that this is high enough for an efficient production of s-process elements to occur by starting from lighter seeds $(\mathrm{C}-\mathrm{Ne})$, even in the complete absence of ironpeak elements.

The calculations of the $1 M_{\odot}$ metal-free model presented in this paper (model M1) show that low-mass stars can also produce light elements that might act as seeds of s-process nucleosynthesis. This occurs in two steps. First, the interior temperature is high enough to start helium-burning and produce carbon during the transition from the subgiant to the red giant phase. Once the carbon mass-fraction is $X_{\mathrm{c}} \sim 10^{-11}$, the CNO cycle is ignited, and a small thermal runaway occurs due to the sudden increase in the efficiency of the CNO burning (Weiss et al. 2000). Later, neutrons are captured by ${ }^{12} \mathrm{C}$ and ${ }^{16} \mathrm{O}$ creating $\mathrm{Ne}$ and $\mathrm{Na}$ isotopes, in the PIE.

In model M1, however, the HeCZ splits before s-process elements can be produced by neutron capture onto the light seeds. Since the splitting of the $\mathrm{HeCZ}$ is followed by an abrupt decrease in the availability of neutrons (Figure 4, top panel), s-process elements are not formed despite reaching a high neutron density $\left(n_{\mathrm{n}}>10^{13} \mathrm{~cm}^{-3}\right)$. The neutron exposure achieved at the position of maximum energy release by He-burning is $\sim 25 \mathrm{mb}^{-1}$, which would be sufficient to produce s-process elements if the neutrons were captured in a radiative environment, as it happens during the TDU in AGB stars (Goriely \& Siess 2001). 


\section{Discussion}

The results presented in the previous sections show that the production of s-process elements in the models is mainly determined by the time spanned between the start of the PIE and the splitting of the convective zone $\left(\Delta t_{\mathrm{PIE}}\right)$ and the time evolution of the neutron flux. It is in this context that we discuss our results and compare them to those from other authors in this section.

Fujimoto et al. (2000) proposed a general picture for the enrichment of CNO and, potentially, s-process elements in EMP stars. Although stars with mass $M<1.2 M_{\odot}$ and metallicity $[\mathrm{Fe} / \mathrm{H}]<-4$ experience the PIE during the core He-flash in their models, the splitting timescale for the $\mathrm{HeCZ}$ is so short that there is no s-process enhancement in the surface. In their models, that are based on those by Hollowell et al. (1990), they find $\Delta t_{\mathrm{PIE}} \approx 2.5 \times 10^{-3} \mathrm{yr}$ during the core He-flash. In more recent results by the same group (Suda \& Fujimoto 2010), the picture remains similar to the one proposed by Fujimoto et al. (2000), although there is no available information about $\Delta t_{\mathrm{PIE}}$. On the other hand, Campbell et al. (2010) found a large s-process production during the PIE of a star $M=1 M_{\odot}$ and a metallicity of $[\mathrm{Fe} / \mathrm{H}]=-6.5$. Our models for the core He-flash agree with results by Campbell et al. (2010) in terms of the existence of s-process production during this evolutionary stage. The level of production and of surface enrichment are, however, quantitatively different.

It should be stated that an important difference among calculations by different authors relates to the treatment of mixing during the PIE. As described before, our calculations with GARSTEC use a diffusive approach for convective mixing to account for the competition between mixing and nuclear burning. This is similar to the scheme used by Campbell et al. (2010). On the other hand, PIE models computed by Fujimoto et al. (2000) and Suda \& Fujimoto (2010) assume homogeneous (instant) mixing down to a depth in the $\mathrm{HeCZ}$ determined by equating the convective turn-over and proton captures by ${ }^{12} \mathrm{C}$ timescales. Is it this less physical approach at the root of the qualitative differences in the results regarding s-process nucleosynthesis?

In our models, we do not find the neutron superburst present in the calculations of Campbell et al. (2010), where neutron density values of $\sim 10^{15} \mathrm{~cm}^{-3}$ are reached at the beginning of the PIE, which leads to the immediate production of large amounts of s-process elements. Our results show that the neutron density increases smoothly up to values above $10^{14} \mathrm{~cm}^{-3}$ (see Fig. 4) as the ingestion of protons progresses. This results in a slower production of the s-process in the $\mathrm{HeCZ}$ than the one found by Campbell et al. (2010). The larger size of our convective zone also contributes to effectively slowing down the production of s-process elements. The combination of a slower production and a shorter timescale for the splitting leads to surface abundances which are lower than those reported by Campbell et al. (2010). For instance, their Sr surface abundance is more than 2 dex larger than ours (Table 2). However, a direct comparison with our results is difficult, because production of s-process elements also depends on average properties of the neutron exposure in the convective shell, and no information is provided by Campbell et al. (2010). We also note here that Campbell et al. (2010) had to extrapolate their results because of numerical problems which did not allow them to follow the dredge-up of processed material into the stellar envelope. In contrast, our results are based on full models that follow the enrichment of the stellar surface as a consequence of the merging between the $\mathrm{HCZ}$ and the convective envelope following the PIE in detail. Although we do not believe the difference in the final enrichment is caused because
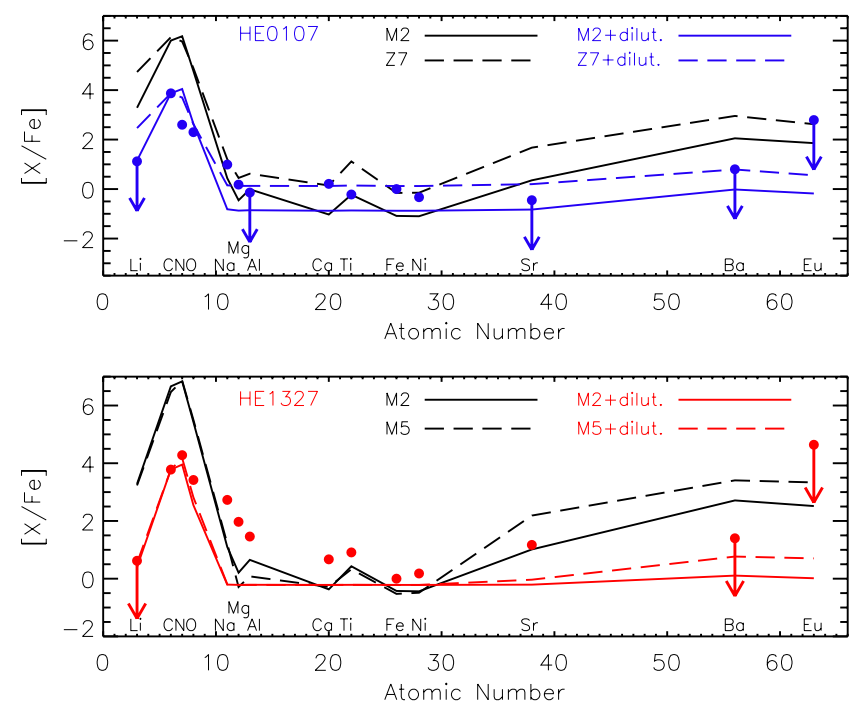

Fig. 8. Top panel: abundance pattern of HE0107-5240 (blue filled circles, from Christlieb et al. 2004; and Bessell et al. 2004). Black solid and dashed lines represent the surface abundance of models M2 and Z7 (see Sect. 6 for more details on this model) respectively. Blue lines show the abundance patterns after dilution by factors of $f_{\mathrm{D}}=7.4 \times 10^{-3}$ and $5.4 \times 10^{-3}$ (models M2 and Z7 respectively), which are derived by matching the observed carbon abundance. Arrows indicate upper limits. $\mathrm{Li}$ abundance is given as $\log \epsilon(\mathrm{Li})=\log \left(\mathrm{N}_{\mathrm{Li}} / \mathrm{N}_{\mathrm{H}}\right)+12$. The zero point on the $y$-axis corresponds to $[\mathrm{Fe} / \mathrm{H}]=-5.3$. Bottom panel: same as above but for HE-1327-2326 (red filled circles, data from Frebel et al. 2008). For this star, the models shown are M2 and M5. Abundance patterns after dilution $\left(f_{\mathrm{D}}=1.3 \times 10^{-3}\right.$ and $2.1 \times 10^{-3}$ for M2 and M5 respectively) are shown in red solid and dashed lines, as indicated in the plot. The zero point of the $y$-axis corresponds to $[\mathrm{Fe} / \mathrm{H}]=-5.96$.

of extrapolations done by Campbell et al. (2010) (which is based on reasonable assumptions), it is clear our calculations represent a qualitative step forward in modeling the PIE and in the subsequent enrichment of the stellar envelopes.

We have compared our models with the hyper metal-poor (HMP) stars, HE0107-5240 and HE1327-2326. Agreeing with previous works, the first conclusion is that the self-pollution scenario cannot explain the abundance patterns observed in HE0107-5240 (Picardi et al. 2004; Suda et al. 2004; Weiss et al. 2004; Campbell et al. 2010). Models produce $\sim 3$ dex more $[\mathrm{C} / \mathrm{Fe}]$ and at least 2 dex more $[\mathrm{Sr} / \mathrm{Fe}]$ and $[\mathrm{Ba} / \mathrm{Fe}]$ than observed in HE0107-5240. This is shown in the top panel of Fig. 8, where abundances derived from observations are shown in blue circles. Surface abundances of model M2 after dredgeup are represented by the black solid line. Note that model abundances have been shifted to be on the scale determined by $[\mathrm{Fe} / \mathrm{H}]=-5.3$, corresponding to this star, which is about one order of magnitude larger than model M2. Additionally, we have computed model Z7 with a solar scaled metallicity $Z=10^{-7}$, which closely matches $[\mathrm{Fe} / \mathrm{H}]$ value for HE0107-5240. Results are shown in a dashed black line and lead to similar conclusions as for model M2. The self-pollution scenario is not even applicable to HE1327-2326, because this star is not a giant and has not undergone the He-core flash yet. In the binary scenario, on the other hand, there is no restriction to the evolutionary stage of the observed star, and we can compare our models to both stars. In this scenario, material is transferred from the primary star (represented here by our models) and diluted in the envelope of the companion (observed) star. The dilution factor, $f_{\mathrm{D}}$, represents the fractional mass of accreted material with respect to the mass 
of the accreting star over which the polluting material is mixed. Simply,

$\epsilon_{i}^{*}=\frac{\epsilon_{i}^{\mathrm{ini}}+f_{\mathrm{D}} \epsilon_{i}^{\mathrm{PIE}}}{1+f_{\mathrm{D}}}$,

where $\epsilon_{i}^{*}$ is the observed abundance, $\epsilon_{i}^{\text {ini }}$ the initial model abundance, and $\epsilon_{i}^{\mathrm{PIE}}$ the abundance after the dredge up that follows the occurrence of the $\mathrm{PIE}^{3}$ for a reference element $i$. Here, we use carbon as the reference element to compute $f_{\mathrm{D}}$ and assume the initial carbon abundance is the same in both binary components.

Let us first consider HE0107-5240. For models M2 and Z7 shown in top panel of Fig. 8, the dilution factors are $f_{\mathrm{D}}=7.4 \times 10^{-3}$ and $f_{\mathrm{D}}=5.4 \times 10^{-3}$, respectively. The abundance patterns after applying dilution are represented by a blue solid (M2) and dashed lines (Z7). The vertical displacement between the models for the $\mathrm{Fe}$ abundance simply reflects the different initial metallicities of the models. The almost constant difference between models M2 and Z7 for all elements higher than Al are evidence that production of these elements scale almost linearly with the initial composition of the models. After including dilution, models $\mathrm{M} 2$ and $\mathrm{Z7}$, produce oxygen that agrees with the observed values within a factor of two, while $\mathrm{N}$ is overproduced by a factor of $\sim 27$ (M2) and $\sim 12$ (Z7). For elements observed in this star in the range between $\mathrm{Mg}$ and $\mathrm{Ni}$, there is a very modest overproduction of elements in our models with respect to Fe. After dilution, the resulting abundance pattern for these elements shown in Fig. 8 is almost flat and reflects mostly the initial solar abundance pattern of the models. The dilution factor, determined by the extremely large carbon enhancement, is too small to have a large impact on these elements. For the s-process elements, $\mathrm{Sr}$ and $\mathrm{Ba}$, and $\mathrm{Eu}$ where observations only allow an upper limit abundance determination, the situation is similar. Although these elements are strongly overproduced during the PIE (see Table 2 and black lines in Fig. 8), abundances after dilution show a pattern very close to solar, which reflect the initial relative distribution of abundances in the models. In this regard, results shown in Fig. 8 can be taken as an upper limit of the abundances that the models presented in this work and the pollution scenario can lead to.

In the case of HE1327-2326, we adopt $[\mathrm{Fe} / \mathrm{H}]=-5.96$ (Frebel et al. 2008), which is close the Fe abundance in our reference model M2. In the bottom panel of Fig. 8, results for model $\mathrm{M} 2$ before and after dilution $\left(f_{\mathrm{D}}=1.3 \times 10^{-3}\right)$ are compared to the observed abundances. To show the impact of uncertainties in the modeling of the PIE, we also show results for M5 $\left(f_{\mathrm{D}}=2.1 \times 10^{-3}\right)$, which is the model with very large overshooting. There is a reasonably good agreement for $\mathrm{N}$ and $\mathrm{O}$ abundances within a factor of 2 for $\mathrm{N}$ and 4 for $\mathrm{O}$. For all other elements, overproduction factors are totally damped by the small values of the dilution factors, except for Ba in model M5.

One interesting signature of the PIE is the ${ }^{7} \mathrm{Li}$ production (Iwamoto et al. 2004; Cristallo et al. 2009). Unfortunately, observations only allow an upper limit determination of its abundance in these two stars. For HE1327-2326 in particular, our models lead to a value that agrees very well with the observational upper limit. For HE0107-5240, on the other hand, model Z7 overproduces Li by more than one order of magnitude. Our models do not account for any possible destruction of $\mathrm{Li}$ after mass transfer.

In our simple dilution model, we have assumed that mass was transferred before the star entered the AGB phase. Lowmass stars that suffered PIE during the core He-flash undergo a normal AGB evolution. Therefore, the production of

\footnotetext{
$3 \log \epsilon_{\mathrm{i}}=\log N_{\mathrm{i}} / N_{\mathrm{H}}+12$.
}

s-process elements should happen in a radiative environment if a ${ }^{13} \mathrm{C}$ pocket is formed. We might speculate on the possible consequence if the primary had not transferred mass to the companion star right after the PIE but rather during or after its AGB evolution. The main products carried to the surface after the TDU are carbon and s-process elements. Hence, the primary would be even more enriched in these elements than right after the PIE. Nitrogen abundance would remain the same, since hot bottom burning does not happen in stars with masses of $M=1 M_{\odot}$ and Li would be depleted during the TDU. In this way, a better match to the nitrogen and the lithium abundances of HE 01075240 could be obtained if the accreted mass is small enough. The ${ }^{12} \mathrm{C} /{ }^{13} \mathrm{C}$ ratio would also increase during the AGB evolution and could better match the observed lower limit for HE 0107-5240 $\left({ }^{12} \mathrm{C} /{ }^{13} \mathrm{C}>50\right)$.

An important finding of this work is that only elements lighter than $\mathrm{Fe}$ are synthesized in the $Z=0$ model. Our results show that the splitting time $\Delta t_{\mathrm{PIE}}$ of the HeCZ is too short, and not enough neutrons are produced to go beyond Fe. In the viewpoint of the binary pollution scenario, this result does not support a low-mass zero metallicity star as the polluting companion of the two HMP stars that were discussed in this paper, if mass is transferred before it enters the AGB evolution.

In closing, we have done a simple test to determine if the production of s-process elements during the PIE is sensitive to the stellar mass. As stated before, Fujimoto et al. (2000) has found an upper limit of $1.2 M_{\odot}$ for the occurrence of the PIE. On the low-mass range, we have computed the evolution of a $0.82 M_{\odot}$ and $Z=10^{-8}$ model (representative of the lowest stellar mass that has had time to evolve to the core He-flash in the lifetime of the Milky Way) and found that the splitting time is a factor of 5 shorter $\left(\Delta_{\mathrm{PIE}}=0.08 \mathrm{yr}\right)$ in comparison to model M2 but is still more than 30 times larger than models by Hollowell et al. (1990). The neutron flux increases more rapidly than in model M2, and just 0.03 yr after $L_{\max }^{\mathrm{He}}$, the neutron density at the bottom of the $\mathrm{HeCZ}$ is already larger than $10^{14} \mathrm{~cm}^{-3}$. As a result, we find that there is a total neutron exposure at the bottom of the $\mathrm{HeCZ}$ of $130 \mathrm{mb}^{-1}$ and an average neutron exposure over the whole $\mathrm{HeCZ}$ of $30 \mathrm{mb}^{-1}$ that is associated with the PIE. We have not performed post-processing nucleosynthesis for this model. However, the abundance pattern during the $\mathrm{HeCZ}$ is very similar to model M2 (and common to all models of equal metallicity undergoing the core He-flash), and the neutron exposure is very large and within a factor of 2 of model M2. For these reasons and the results presented in this work, we conclude that s-process elements are efficiently produced with a qualitatively similar abundance pattern in EMP stars in the low-mass range of stars suffering a PIE during the core He-flash, that is for stellar masses smaller than about $1.2 M_{\odot}$.

\section{Conclusions}

We have performed evolutionary calculations for low-mass EMP and zero metallicity stars. Our models follow the PIE during the core He-flash and the subsequent hydrogen flash. We have then used these calculations as input to a post-processing unit, where we have performed calculations of s-process nucleosynthesis. A comparison with and among similar calculations found in the literature shows that the location at which the He-flash occurs varies among different stellar codes (varying from $0.15 M_{\odot}$ (Schlattl et al. 2001), $\sim 0.24 M_{\odot}$ (this paper) to $0.41 M_{\odot}($ Hollowell et al. 1990)). This is indicative of the uncertainties in stellar modeling and is likely to impact results of the PIE modeling. 
The production of s-process elements and subsequent surface enrichment during the PIE in EMP low-mass stars depends strongly on the efficiency of the convective mixing and, in particular, on the $\Delta t_{\mathrm{PIE}}$, which is the time elapsed between the onset of the hydrogen mixing and the splitting of the convective zone. We do find large production of s-process elements in our models. The surface abundances after dredge- up are, however, about 2 dex smaller than those reported by Campbell et al. (2010). This is likely the result of the difference in the neutron density history between our models and those by Campbell et al. (2010); we do not find the neutron superburst reported by these authors. A detailed comparison with Campbell et al. (2010) is difficult. Although they report a total neutron exposure of $287 \mathrm{mb}^{-1}$, which is close to our results for models M2 and M3, the PIE takes place in a convective region, and neutron production is a strong function of temperature, as it is determined by both the producion and burning of ${ }^{13} \mathrm{C}$. The actual production of s-process elements, therefore, does not depend on the maximum neutron exposure but on its averaged value. There is no information provided on this regard by Campbell et al. (2010), nor is there information on how the convective region has been treated in their post-processing code. In more detail, our models produce 2-3 dex less for the first and second peak sprocess elements, whereas $\mathrm{Pb}$ production is smaller in our models by 1.3 dex. On the other hand, our results qualitatively agree that s-process production happens during the PIE in the coreHe flash phase, which is in contrast with (Fujimoto et al. 2000; Suda \& Fujimoto 2010) results that found no relevant s-process production during the PIE.

Our models produce $\mathrm{C}, \mathrm{N}$, and $\mathrm{O}$ surface abundances $2-3$ dex larger than the abundance values observed in the HMP stars. The high CNO abundances in our models disfavor the selfenrichment scenario to explain the EMP abundance patterns, as other works on the topic have shown before. In the viewpoint of the mass-transfer scenario, our models support the idea that a low-mass star can be the donor star because the dilution of the transferred material occurs in the secondary star envelope. Our estimates of the dilution factors during the accretion process show that the secondary needs to accrete a mass equal to only a few parts per thousand of its own envelope mass in order to match the observed carbon abundances. The small dilution factors, on the other hand, makes it difficult to enhance the s-process abundances of the accreting star for the overproduction levels we find and report in Table 2 .

Based on our results and agreeing qualitatively with those from Campbell et al. (2010), we conclude that stars with masses of $M \leq 1 M_{\odot}$ cannot be excluded as the binary companions of the two most iron deficient stars yet observed. In addition, the latest findings by Caffau et al. $(2011,2012)$ push the lowest metallicity at which low mass stars can form, increasing the need of detailed models for this class of stars. All these results reinforce the necessity of detailed studies of the PIE, both with hydrodynamic simulations and stellar evolutionary models to achieve a more realistic picture of the its properties.

Acknowledgements. This work is part of the Ph.D. thesis of Monique Alves Cruz and has been funded by the IMPRS fellowship. This research was supported by the DFG cluster of excellence "Origin and Structure of the Universe". Aldo Serenelli is partially supported by the European Union International Reintegration Grant PIRG-GA-2009-247732 and the MICINN grant AYA201124704. M.A.C. would like to thank N.M. Fernandes for the careful reading of the manuscript, and Silvia Rossi for providing her with a nice work space for the past months. We acknowledge the numerous comments by an anonymous referee that have helped us improved presentation of results.

\section{References}

Abel, T., Bryan, G. L., \& Norman, M. L. 2002, Science, 295, 93

Adelberger, E. G., Garía, A., Robertson, R., et al. 2011, RvMP, 83, 195

Althaus, L. G., Serenelli, A. M., Panei, J. A., et al. 2005, A\&A, 435, 631

Angulo, C., Arnould, M., Rayet, M., et al. 1999, Nucl. Phys. A, 656, 3

Aoki, W., Norris, J. E., Ryan, S. G., Beers, T. C., \& Ando, H. 2002, ApJ, 567, 1166

Beers, T. C., \& Christlieb, N. 2005, ARA\&A, 43, 531

Bessell, M. S., Christlieb, N., \& Gustafsson, B. 2004, ApJ, 612, L61

Bromm, V., \& Loeb, A. 2003, Nature, 425, 812

Bromm, V., \& Loeb, A. 2004, New Astron., 9, 353

Bromm, V., Coppi, P. S., \& Larson, R. B. 2002, ApJ, 564, 23

Caffau, E., Bonifacio, P., François, P., et al. 2011, Nature, 477, 67

Caffau, E., Bonifacio, P., François, P., et al. 2012, A\&A, 542, A51

Campbell, S. W., \& Lattanzio, J. C. 2008, A\&A, 490, 769

Campbell, S. W., Lugaro, M., \& Karakas, A. I. 2010, A\&A, 522, A6

Chieffi, A., Domínguez, I., Limongi, M., \& Straniero, O. 2001, ApJ, 554, 1159

Christlieb, N., Gustafsson, B., Korn, A. J., et al. 2004, ApJ, 603, 708

Cristallo, S., Piersanti, L., Straniero, O., et al. 2009, PASA, 26, 139

Cyburt, R., Amthot, A. M., Ferguson, R., et al. 2010, ApJS, 189, 240

Dillmann, I., Heil, M., Plag, R., Rauscher, T., \& Thielemann, F. K. 2006, AIP Conf. Ser., 819, 123

Ferguson, J. W., Alexander, D. R., Allard, F., et al. 2005, ApJ, 623, 585

Frebel, A., Collet, R., Eriksson, K., Christlieb, N., \& Aoki, W. 2008, ApJ, 684, 588

Freytag, B., Ludwig, H., \& Steffen, M. 1996, A\&A, 313, 497

Fujimoto, M. Y., Iben, I. J., \& Hollowell, D. 1990, ApJ, 349, 580

Fujimoto, M. Y., Ikeda, Y., \& Iben, I. J. 2000, ApJ, 529, L25

Goriely, S., \& Siess, L. 2001, A\&A, 378, 25

Greif, T. H., Springel, V., White, S. D. M., et al. 2011, ApJ, 737, 75

Grevesse, N., \& Sauval, A. J. 1998, Space Sci. Rev., 85, 161

Hale, S. E., Champagne, A. E., Iliadis, C., et al. 2002, Phys. Rev. C, 65, 015801

Hale, S. E., Champagne, A. E., Iliadis, C., et al. 2004, Phys. Rev. C, 70, 045802

Herwig, F. 2001, ApJ, 554, L71

Herwig, F., Langer, N., \& Lugaro, M. 2003, ApJ, 593, 1056

Herwig, F., Pignatari, M., Woodward, P. R., et al. 2011, ApJ, 727, 89

Hollowell, D., Iben, I. J., \& Fujimoto, M. Y. 1990, ApJ, 351, 245

Iglesias, C. A., \& Rogers, F. J. 1996, ApJ, 464, 943

Itoh, N., Mitake, S., Iyetomi, H., \& Ichimaru, S. 1983, ApJ, 273, 774

Iwamoto, N., Kajino, T., Mathews, G. J., Fujimoto, M. Y., \& Aoki, W. 2004, ApJ, 602, 377

Jaeger, M., Kunz, R., Mayer, A., et al. 2001, Phys. Rev. Lett., 87, 202501

Jorissen, A., \& Arnould, M. 1989, A\&A, 221, 161

Kubono, S., Abe, K., Kato, S., et al. 2003, Phys. Rev. Lett., 90, 062501

Kunz, R., Jaeger, M., Mayer, A., et al. 2002, ApJ, 567, 643

Lucatello, S., Tsangarides, S., Beers, T. C., et al. 2005, ApJ, 625, 825

Luck, R. E., \& Bond. 1991, ApJS, 77, 515

Magic, Z., Serenelli, A., Weiss, A., \& Chaboyer, B. 2010, ApJ, 718, 1378

Masseron, T., Johnson, J. A., Plez, B., et al. 2010, A\&A, 509, A93

Moazen, B. H., Bardayan, J. C., Blackmon, K. Y., Chae, K. Y., et al. 2007, Phys. Rev. C, 75, 065801

O'shea, B. W., \& Norman, M. L. 2007, ApJ, 654, 66

Picardi, I., Chieffi, A., Limongi, M., et al. 2004, ApJ, 609, 1035

Reimers, D. 1975, Mem. Soc. Roy. Sci. Liège, 8, 369

Rogers, F. J., Swenson, F. J., \& Iglesias, C. A. 1996, ApJ, 456, 902

Rossi, S., Beers, T. C., \& Sneden, C. 1999, ASPC, 165, 264

Schlattl, H. \& Weiss, A. 1999, A\&A, 347, 272

Schlattl, H., Cassisi, S., Salaris, M., \& Weiss, A. 2001, ApJ, 559, 1082

Schneider, R., Ferrara, A., Salvaterra, R., Omukai, K., \& Bromm, V. 2003, Nature, 422, 869

Serenelli, A. 2006, in Chemical Abundances and Mixing in Stars in the Milky Way and its Satellites, eds. S. Randich, \& L. Pasquini (Springer-Verlag), 322 Stancliffe, R. J., Dearborn, D. P., Lattanzio, J., Heap, S. A., \& Campbell, S. W. 2011, ApJ, 742, 121

Suda, T., \& Fujimoto, M. Y. 2010, MNRAS, 405, 177

Suda, T., Aikawa, M., Machida, M. N., Fujimoto, M. Y., \& Iben, J. I. 2004, ApJ, 611,476

Suda, T., Fujimoto, M. Y., \& Itoh, N. 2007, ApJ, 667, 1206

Suda, T., Yamada, S., Katsuta, Y., et al. 2011, MNRAS, 412, 843

Takahashi, K., \& Yokoi, K. 1987, At. Data Nucl. Data Tables, 36, 375

Tomkin, J., Lamnert, D. L., Edvardsson, B., Gustafsson, B., \& Nissen, P. E. 1989, A\&A, 219, L15

Weiss, A., \& Ferguson, J. W. 2009, A\&A, 508, 1343

Weiss, A., \& Schlattl, H. 2008, Ap\&SS, 623, 99

Weiss, A., Cassisi, S., Schlattl, H., \& Salaris, M. 2000, ApJ, 533, 413

Weiss, A., Schlattl, H., Salaris, M., \& Cassisi, S. 2004, A\&A, 422, 217

Yoshida, N., Omukai, K., \& Hernquist, L. 2008, Science, 321, 669 